\title{
Lecithin/sphingomyelin ratio in hypopharyngeal aspirate of newborn infants
}

\author{
PETER A. BARR, PATRICIA A. JENKINS, and J. DAVID BAUM \\ From the Department of Paediatrics, University of Oxford, John Radcliffe Hospital
}

\begin{abstract}
Barr, P. A., Jenkins, P. A., and Baum, J. D. (1975). Archives of Disease in Childhood, 50, 856. Lecithin/sphingomyelin ratio in hypopharyngeal aspirate of newborn infants. The lecithin/sphingomyelin $(\mathrm{L} / \mathrm{S})$ ratio was measured in the hypopharyngeal aspirate of 41 newborn infants. In 20 without respiratory distress the ratio within 24 hours from birth ranged from 1.5 to 5.8 with a mean value of 3.3 . In 6 with 'transient tachypnoea of the newborn' the ratio ranged from $2 \cdot 0$ to 6.7 with a mean value of $3 \cdot 2$. In 15 infants with idiopathic respiratory distress syndrome (RDS) the ratio ranged from 0.9 to $2 \cdot 1$ with a mean value of 1.4 . In 14 of these infants the ratio within 24 hours from birth was 1.7 or less. One infant with a higher ratio and severe RDS is described in detail.

Serial hypopharyngeal aspirate $\mathrm{L} / \mathrm{S}$ ratios were determined in the infants with RDS. There was a possible trend toward the earlier attainment of a ratio of 2.0 in infants who received continuous distending airway pressure compared with infants who did not.
\end{abstract}

The lungs of newborn infants with the idiopathic respiratory distress syndrome (RDS) lack the surface activity necessary for maintaining alveolar stability (Avery and Mead, 1959). The phospholipid dipalmitoyl lecithin, which is primarily responsible for the stability of the mature lung, is greatly reduced in amount in the lungs of infants dying with RDS (Adams et al., 1965). Lungs from infants dying in the neonatal period after recovery from RDS have been shown by Brumley, Hodson, and Avery (1967) to have normal surface tension forces and normal saturated lecithin concentrations.

Gluck et al. (1971) showed that the amount of amniotic fluid surface-active lecithin, expressed semiquantitatively as the lecithin/sphingomyelin $(\mathrm{L} / \mathrm{S})$ ratio, accurately predicts fetal lung maturity and thus the likelihood of RDS developing. The $\mathrm{L} / \mathrm{S}$ ratio has also been measured in the tracheal (Kanto et al., 1974b; Kanto, Borer, and Roloff, 1974a; Blumenfeld, Driscoll, and James, 1974), gastric (Borer et al., 1974), and hypopharyngeal (Blumenfeld et al., 1974; Fox, Sarkozi, and Rosen feld, 1974) aspirates of newborn infants. Fox et al. (1974) concluded that neonatal pulmonary biochemical maturity could be assessed by the

Received 20 March 1975.
L/S ratio in the hypopharyngeal aspirate. But Blumenfeld $e t$ al. (1974), in a similar study, were unable to confirm these findings.

In this paper we present our experience of the $\mathrm{L} / \mathrm{S}$ ratio in the hypopharyngeal aspirate and its relationship to the clinical course in newborn infants.

\section{Patients and methods}

Respiratory distress was defined as a respiratory rate of more than $60 / \mathrm{min}$ within four hours from birth, sustained for more than four hours, and accompanied by expiratory grunting or inspiratory retractions or both. In addition, the infants with respiratory distress in this study required extra ambient oxygen either to relieve cyanosis or to maintain the arterial oxygen tension at or above $60 \mathrm{mmHg}$.

A total of 41 infants were studied and they were divided into three groups (Table I). Group 1 comprised 20 infants without respiratory distress. Group 2 comprised 6 infants with respiratory distress which was thought not to be RDS. Group 3 comprised 15 infants with RDS. The chest radiographs of the infants with respiratory distress were reviewed by a paediatric radiologist who had no knowledge of the patients.

Five of the 6 infants in the group 2 (considered not to have RDS) had the clinical course and chest radiographic signs of 'transient tachypnoea of the newborn' 
TABLE I

Clinical data in 41 newborn infants subgrouped according to presence or absence of respiratory distress

\begin{tabular}{|c|c|c|c|}
\hline & Distress absent & TTN & RDS \\
\hline $\begin{array}{l}\text { No. of infants } \\
\text { Birthweight }(g)^{\star} \\
\text { Gestation }(w)^{\star}\end{array}$ & $\begin{array}{c}20 \\
2894 \\
1380-4410 \\
37 \cdot 5 \\
33-40\end{array}$ & $\begin{array}{c}6 \\
2953 \\
1800-4020 \\
36 \cdot 7 \\
23-39\end{array}$ & $\begin{array}{c}15 \\
2355 \\
1200-3900 \\
35 \cdot 7 \\
31-39\end{array}$ \\
\hline
\end{tabular}

*Mean and range.

TTN, transient tachypnoea of the newborn; RDS, idiopathic respiratory distress syndrome.

(Avery, Gatewood, and Brumley, 1966). Their respiratory distress was mild. The highest inspired oxygen concentration needed by any of them was $40 \%$ and in all of them the tachypnoea had subsided within 60 hours from birth. The chest $x$-rays showed prominent linear markings radiating from the lung hila. In 2 infants this was accompanied by slight cardiomegaly and in 2 others by areas of decreased radiolucency. Overdistension of the lung fields was present in only one infant. The remaining infant in group 2 had a normal chest $x$-ray but in all other respects resembled the other 5 .

The infants in group 3 (considered to have RDS) had respiratory distress which lasted longer than 24 hours and a chest $x$-ray with a reticulogranular appearance.

The aim in managing the infants with RDS was to keep the arterial oxygen tension between 60 and 90 $\mathrm{mmHg}$ with an inspired oxygen concentration of $60 \%$ or less. If this was possible without assistance to ventilation the RDS was regarded as mild (7 infants). If continuous positive airway pressure (CPAP) was required the RDS was regarded as moderate ( 5 infants). If intermittent positive pressure ventilation (IPPV) with positive end-expiratory pressure (PEEP) was required the RDS was regarded as severe (3 infants). None of the infants died.

In each of the 41 infants a hypopharyngeal aspirate was obtained within 24 hours from birth. Twenty of the samples were obtained within 8 hours, 11 between 8 and 16 hours, and 10 between 16 and 24 hours. In the infants with respiratory distress additional samples were obtained beyond the first 24 hours from birth. The secretions were aspirated with a Macrae mucus extractor.* The aspirate was flushed from the catheter with $0.5 \mathrm{ml}$ of $0.9 \%$ saline and the specimen stored at $-20^{\circ} \mathrm{C}$ until analysis. Samples containing either blood or meconium were discarded as unsuitable.

Biochemical analyses. The method used for extracting phospholipids from the aspirate was similar to that described for amniotic fluid by Borer and Gluck (1971). The aspirate was, when necessary, made up to a volume of $1.0 \mathrm{ml}$ by adding $0.9 \%$ saline. One $\mathrm{ml}$ of uncentrifuged hypopharyngeal aspirate was added to $1.0 \mathrm{ml}$ of methanol and mechanically mixed for 15

^Warne Surgical Products Ltd., Andover, Hants., England. seconds. Two $\mathrm{ml}$ of chloroform were added and the mixture mechanically mixed for a further 30 seconds. The resulting emulsion was placed in ice for 5 minutes and then centrifuged at 3000 r.p.m. for 10 minutes. The chloroform layer at the bottom was carefully removed and evaporated to dryness under a stream of nitrogen in a water bath at $60^{\circ} \mathrm{C}$. Ten drops of ice-cold acetone were added to precipitate the surface-active lecithin (Gluck et al., 1967). The acetone was decanted and the precipitate dried under a stream of nitrogen. The precipitate was dissolved in $10 \mu \mathrm{l}$ of chloroform and spotted on to a commercially prepared thin layer silica gel chromatographic strip (No. 6061)* cut to measure $7.5 \mathrm{~cm}$ by $2.5 \mathrm{~cm}$. The chromatogram was developed in a chromatogram tank using freshly prepared solvent of methanol, chloroform, and water (v:v:v 25:65:4). The solvent was allowed to run within $1.0 \mathrm{~cm}$ of the top of the strip and then the chromatogram was dried in air.

Two methods were used for staining the phospholipids. In the first 14 patients a total of 28 chromatograms were stained with buffered bromothymol blue (bromothymol blue sodium salt $50 \mathrm{mg}, 1.0 \mathrm{~N}$ sodium hydroxide $8.0 \mathrm{ml}$, boric acid powder $1.25 \mathrm{~g}$, and distilled water $120 \mathrm{ml}$ ) (Borer and Gluck, 1971). The chromatograms were dipped in the bromothymol blue solution for 10 seconds and then oven-dried at $70^{\circ} \mathrm{C}$. The phospholipid spots were visualized by exposing the chromatograms to ammonia vapour which coloured the lecithin and sphingomyelin spots dark blue against a light-blue background. This produced only a transient colouring of the phospholipids and therefore measurement of the spots by reflectance densitometry was not possible.

In the remaining 27 patients a total of 72 chromatograms were stained with freshly prepared phosphomolybdic acid-stannous chloride (Schneider, 1966). The strips were dipped in $2 \%$ phosphomolybdic acid for 30 seconds, washed in running tap water for $\mathbf{3 0}$ minutes, and finally dipped in $4 \%$ anhydrous stannous chloride in hydrochloric acid $1.15 \mathrm{~mol} / 1$ for 30 seconds. The chromatograms were dried in air. This procedure stained the choline fractions of lecithin and sphingomyelin dark blue against a light-blue background. The spots did not fade unless they were exposed to

*Kodak (Eastman), High Holborn, London WC1. 
direct sunlight and therefore were readily measurable by reflectance densitometry.

The L/S ratio of the chromatograms stained with bromothymol blue was calculated by dividing the area of the lecithin spot by the area of the sphingomyelin spot. The area of each spot was calculated by multiplying the maximum width of the spot by its maximum length as measured by calipers to the nearest $0.5 \mathrm{~mm}$. The L/S ratio of the chromatograms stained with phosphomolybdic acid-stannous chloride was calculated from the areas of the respective spots, as described above, and also from reflectance densitometric measurements ${ }^{\star}$ if the spots were dense enough.

Duplicate measurements were not made on the hypopharangeal aspirates because of the small volume of the samples. However, the reproducibility of the method was examined on an amniotic fluid sample and the coefficient of variation was $3 \cdot 7 \%$.

Wagstaff, Whyley, and Freedman (1974) have shown that very similer spot area measurements are obtained when known amounts of lecithin and sphingomyelin are stained with either bromothymol blue or phosphomolybdic acid-stannous chloride. It was not possible to compare the two staining methods on the hypopharyngeal aspirate again because of the small volume of the samples. Instead we compared the two methods on four amniotic fluid samples and four phospholipid standards and found that the two methods did not produce significantly different $\mathrm{L} / \mathrm{S}$ ratios (paired $t$ test, $\mathrm{P}<0.4$ ). Thus in the results recorded below the $\mathrm{L} / \mathrm{S}$ ratios from area measurements of chromatograms stained with bromothymol blue or phosphomolybdic acid-stannous chloride are not considered separately.

We compared the two methods of measuring the spots on the chromatograms. The L/S ratio derived by area measurement was compared with the L/S ratio derived by reflectance densitometry in 64 chromatograms stained with phosphomolybdic acid-stannous chloride (see Fig. 1). There was a close correlation between the two methods within the $L / S$ ratio range which is considered to be of clinical importancenamely, 0.5 to $2 \cdot 5$. In this paper the results given are those derived by area measurement.

To exclude the possibility that milk inadvertantly aspirated from the hypopharynx may have interfered with the results, the milk formulae used in our nursery were analysed by the same procedure of phospholipid extraction, thin layer chromatography, and staining as described above. Faint lecithin and sphingomyelin spots were occasionally seen but the $\mathrm{L} / \mathrm{S}$ ratio in these samples was always less than $1 \cdot 5$.

Using these methods an $\mathrm{L} / \mathrm{S}$ ratio could be obtained within two hours from collecting the sample.

\section{Results}

$L / S$ ratio in infants in group 1 . The mean ratio by area measurement in these control infants

^Chromoscan Mark II double beam recording and integrating densitometer. Joyce, Loebl and Co. Ltd., Gateshead, England.

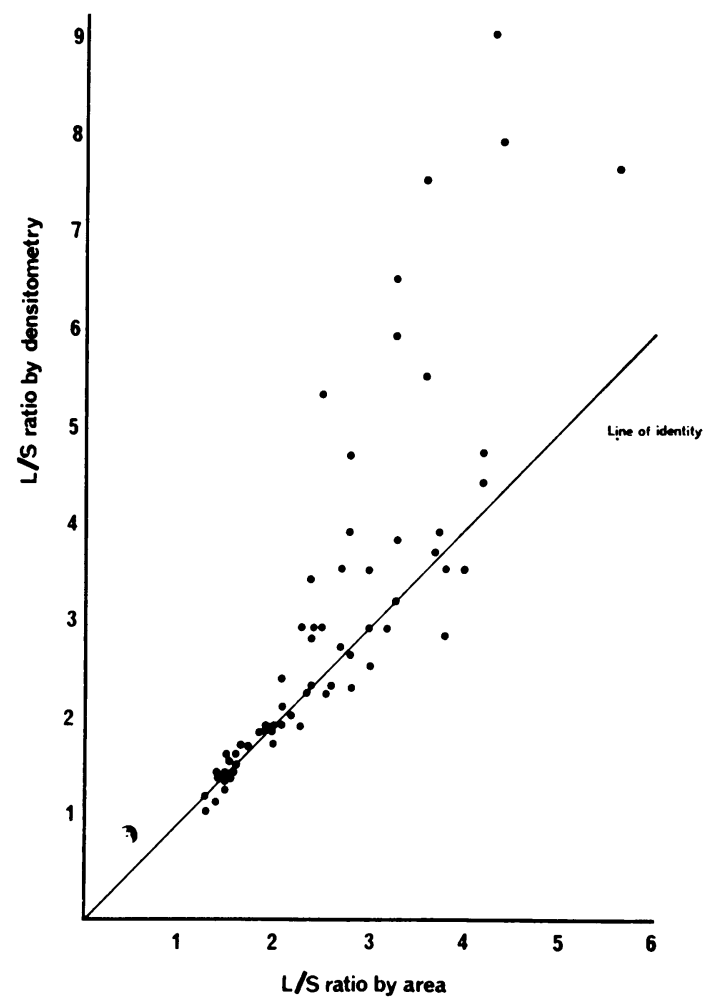

FIG. 1.-Hypopharyngeal aspirate $L / S$ ratio derived from area measurement compared with that from reflectance densitometry in 64 chromatograms stained with phosphomolybdic acid-stannous chloride.

was $3 \cdot 3$ and the range was from $1 \cdot 5$ to $5 \cdot 8$ (Fig. 2). In 17 of the 20 infants in this group the ratio was 2.0 or more. In the 3 remaining infants the ratios of $1.9,1 \cdot 8$, and 1.5 were within what for amniotic fluid has been termed the intermediate range. Fifteen of the 20 samples, including the 3 with ratios of less than $2 \cdot 0$, were obtained within eight hours from birth.

$L / S$ ratio in infants in group 2. The mean ratio by area measurement in the 6 infants in this group was 3.3 and the range was from 2.0 to 6.7 (Fig. 2). Five of the samples in this group were obtained between 8 and 16 hours of age and one within 4 hours from birth. The ratios in this group were not significantly different from those in the control infants (Wilcoxon rank sum test, $\mathbf{P}<0 \cdot 1)$.

$L / S$ ratio in infants in group 3. The mean ratio by area measurement within 24 hours from 


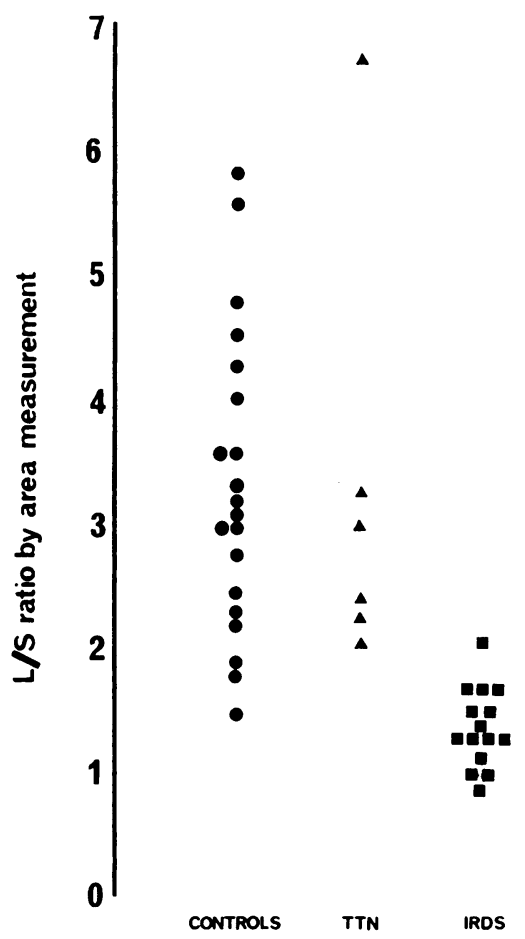

FIG. 2.-Hypopharyngeal aspirate $L / S$ ratio from area measurement within 24 hours from birth in 41 newborn infants. Controls are infants without respiratory distress. $L / S$ ratios in $T T N$ not significantly different from controls. $L / S$ ratios in $R D S$ significantly lower than both controls and TTN $(P<0.001$ by $t$ test $)$.

birth in the 15 infants in this group was $1 \cdot 41$ and the range from 0.9 to $2 \cdot 1$ (Fig. 2). This was significantly lower $(P<0.001)$ than both the group of infants with transient tachypnoea (group 2) and those without respiratory distress (group 1). In 14 of these infants the ratio was 1.7 or less. In 5 infants the ratio was between 1.5 and 1.7 , which is within the intermediate range for amniotic fluid. In these 5 infants the first sample was obtained between 12 and 24 hours of age.

The infant with an hypopharyngeal aspirate L/S ratio of $2 \cdot 1$ presented an unusual clinical history. The pregnancy was terminated at 34 weeks of gestation by emergency caesarean section because of impending eclampsia. The mother had been hypothermic for 30 hours before the operation with a sublingual temperature of less than $36^{\circ} \mathrm{C}$, and for the two hours immediately before delivery it had ranged from $32^{\circ} \mathrm{C}$ to $33^{\circ} \mathrm{C}$. The infant, who weighed $1840 \mathrm{~g}$, was asphyxiated at birth and was given IPPV with $100 \%$ oxygen via an endotracheal tube for 5 minutes. The first rectal tempera- ture, arterial $p \mathrm{H}$, and blood gas tensions, measured at 30 minutes of age were: temperature $33.4{ }^{\circ} \mathrm{C}$, Fio, $0.95 \mathrm{pHa} 7.21, \mathrm{PaO}_{2} 81 \mathrm{mmHg}$, and $\mathrm{PaCO}_{2} 46 \mathrm{mmHg}$. At 4 hours of age the temperature had risen to $36^{\circ} \mathrm{C}$ and in an Fro, of 0.50 the $p \mathrm{Ha}$ was $7 \cdot 20$, the $\mathrm{PaO}_{2} 84$ $\mathrm{mmHg}$, and the Paco, $27 \mathrm{mmHg}$. The infant had the clinical and chest radiographic signs of RDS at the age of 4 hours. He deteriorated over the next 17 hours and IPPV with PEEP was begun at 21 hours of age and was continued for 53 hours, after which CPAP was required for a further 30 hours. The L/S ratio of amniotic fluid obtained at the time of the caesarean section was $1 \cdot 1$ by area measurement and 1.3 by reflectance densitometry. However, serial hypopharyngeal aspirate $L / S$ ratios in the infant were all more than $2 \cdot 0$ (Table II). A tracheal aspirate $\mathrm{L} / \mathrm{S}$ ratio at 50 hours of age was $4 \cdot 3$.

TABLE II

Serial hypopharyngeal aspirate $L / S$ ratios in infant with severe $R D S$

\begin{tabular}{c|c}
\hline Age (h) & L/S ratio \\
\hline 2 & $2 \cdot 1$ \\
9 & $3 \cdot 2$ \\
20 & $2 \cdot 4$ \\
27 & $3 \cdot 7$ \\
45 & $3 \cdot 0$ \\
57 & $2 \cdot 4$ \\
108 & $4 \cdot 2$ \\
\hline
\end{tabular}

Serial hypopharyngeal aspirate $\mathrm{L} / \mathrm{S}$ ratios were determined during the first 96 hours from birth in 13 infants with RDS (Fig. 3). Three of the 6 infants who had not required assisted ventilation (mild RDS) had not reached a ratio of $2 \cdot 0$ by 96 hours of age, whereas only one of the 7 infants who had required assisted ventilation ( 5 with moderate RDS, 2 with severe RDS) had not reached this ratio within the same period.

\section{Discussion}

Gluck et al. (1974) believe that an amniotic fluid L/S ratio of $2 \cdot 0$ or more indicates fetal lung maturity. We have used the methodology of Borer and Gluck (1971) to measure the L/S ratio in the hypopharyngeal aspirate of newborn infants.

There were 15 infants in the present study with RDS and in 14 of them the hypopharyngeal aspirate ratio within 24 hours from birth was less than 1.8. The exception was an infant with severe RDS in whom the ratio was never less than $2 \cdot 0$. This suggests that severe RDS may have occurred in this infant either because of a qualitative deficiency of surfactant or, despite pulmonary biochemical maturity, perhaps because of a quantitative deficiency of surfactant. The perinatal hypothermia 


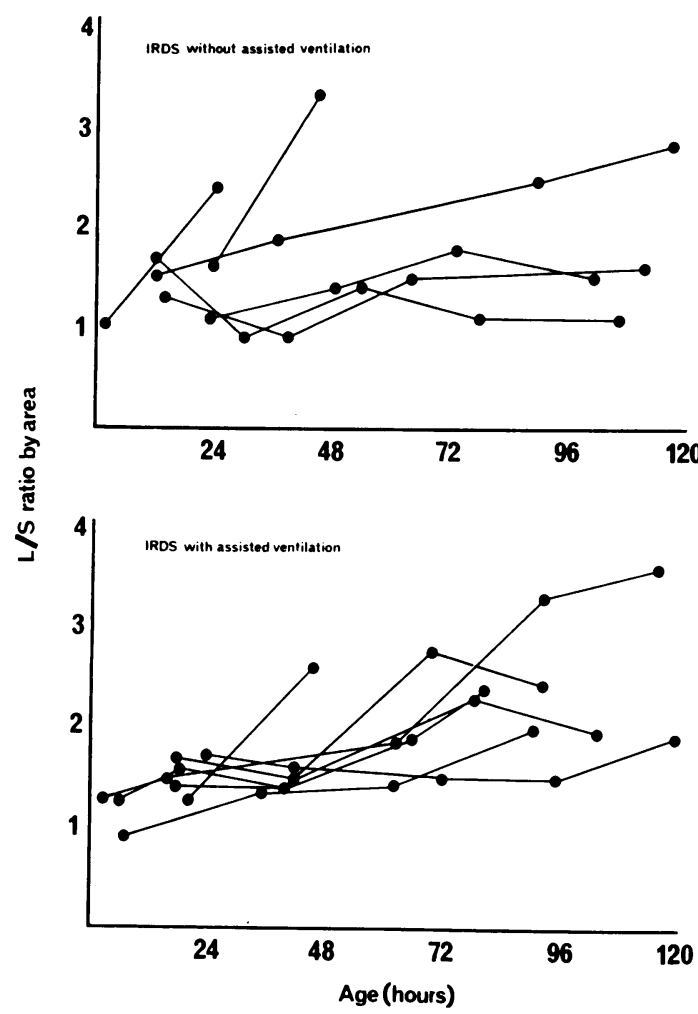

FIG. 3.-Serial hypopharyngeal aspirate $L / S$ ratios in 13 infants with RDS. Upper section shows 6 infants who did not require assisted ventilation (mild $R D S)$; lower section shows 7 infants who required assisted ventilation (moderate or severe RDS). Three infants with mild $R D S$ had not reached $L / S$ ratio of 2.0 by 96 hours of age, whereas only one of the infants with moderate RDS or severe $R D S$ had not reached this $L / S$ ratio in the same period.

may have caused an inefficiency of surfactant, but the effect of hypothermia on the function of surfactant is still not clearly defined (Lempert and Macklem, 1971). Alternatively, the infant's birth asphyxia may have resulted in a primary pulmonary hypoperfusion syndrome (Chu et al., 1967). This case was also of interest because the amniotic fluid $L / S$ ratio was less than $2 \cdot 0$, presumably because of a time lag between the acquisition of pulmonary biochemical maturity, at least quantitatively, in the fetus and its expression in the amniotic fluid as an $\mathrm{L} / \mathrm{S}$ ratio of $2 \cdot 0$ or more.

Our findings agree with those published in abstract form by Borer et al. (1974) for gastric aspirate L/S ratios and Fox et al. (1974) for hypopharyngeal aspirate $L / S$ ratios. They are, however, different from those published by Blumenfeld et al. (1974), who measured the L/S ratio in the tracheal and hypopharyngeal aspirates of 30 newborn infants. Of these 30, 18 had RDS, of whom $13(72 \%)$ had an initial ratio which was mature by the authors' criteria. The difference in results may be partly explained by the fact that the initial sample in $4(22 \%)$ of their infants with RDS was obtained after 24 hours of age. Of the 15 infants with RDS in our study $4(27 \%)$ would have had a ratio of 2.0 or more if the initial sample had been obtained between 24 and 48 hours of age.

Five of the infants with RDS in the present study had hypopharyngeal aspirate $\mathrm{L} / \mathrm{S}$ ratios between 12 and 24 hours of age of from $1 \cdot 5$ to $2 \cdot 0$, which for amniotic fluid has been termed the intermediate range. Three among the 20 infants with no respiratory distress had a ratio of between $1 \cdot 5$ and $2 \cdot 0$. As many as $80 \%$ of infants delivered after the recording of an intermediate amniotic fluid $\mathrm{L} / \mathrm{S}$ ratio are known not to develop RDS (Whitfield and Sproule, 1974). Gluck et al. (1974) suggested that in such cases the amount of acetone precipitable or surfaceactive lecithin is $45 \%$ or more of the total lecithin, and that this itself is an indication of fetal lung maturity. Thus, that an occasional infant without respiratory distress had an hypopharyngeal aspirate $\mathrm{L} / \mathrm{S}$ ratio within the intermediate range is not surprising.

There were 6 infants who had respiratory distress but whose clinical course and chest radiographs were those of 'transient tachypnoea of the newborn.' In all of them the hypopharyngeal aspirate $\mathrm{L} / \mathrm{S}$ ratio in samples obtained within 16 hours from birth was 2.0 or more. That indicates pulmonary biochemical maturity and supports the view that transient tachypnoea of the newborn is a different condition from RDS (Avery et al., 1966). None of these infants had hypopharyngeal aspirate ratios within the intermediate range but presumably these would be found in a larger series, as was the case in 3 of our control infants.

Continuous distending airway pressure (CDAP) is now widely used in the management of RDS (Baum and Roberton, 1974). In most institutions CDAP is started when the inspired oxygen concentration needed to maintain the arterial oxygen tension within normal range is considered to be potentially damaging to the lung. Thus in the present study CDAP was begun when the $\mathrm{PaO}_{2}$ could not be maintained at or above $60 \mathrm{mmHg}$ with an inspired oxygen concentration of $60 \%$ or less. However, the evidence that the early introduction of CDAP shortens the duration (Mockrin 


\section{Lecithin/sphingomyelin ratio in hypopharyngeal aspirate of newborn infants}

and Bancalari, 1974) and decreases the severity (Krouskop, Brown, and Sweet, 1974) of RDS supports the recommendation that CDAP should be started even earlier in the management of RDS (Dunn, 1974). The suggestion from the present study that the $\mathrm{L} / \mathrm{S}$ ratio in the hypopharyngeal aspirate reaches a value of 2.0 earlier in infants receiving CDAP (moderate and severe RDS) than in infants not receiving it (mild RDS) is consistent with the observation that CDAP shortens the duration of RDS.

The evidence that early introduction of CDAP may shorten the duration and decrease the severity of RDS makes it tempting to begin it in an infant with respiratory distress before $\mathrm{RDS}$ is firmly diagnosed or even prophylactically in infants known to be at risk, particularly very immature infants. Nevertheless, in our experience CDAP not only increases arterial oxygen tension but may abolish dyspnoea and significantly modify the chest radiographic appearance. Early introduction of CDAP may therefore obscure the classical diagnostic criteria of RDS. Thus one may on occasion be applying a form of therapy which is inappropriate in an infant who does not have or who will not develop RDS and which is not without serious complications.

It seems from the results of the present study that a hypopharyngeal aspirate $L / S$ ratio of less than 1.5 in an infant with respiratory distress is presumptive evidence of biochemical pulmonary immaturity and by inference of RDS. We believe that an $\mathrm{L} / \mathrm{S}$ ratio of less than 1.5 in an infant with respiratory distress might be a sufficient reason for the early introduction of CDAP even though the reticulogranular appearance of the chest radiograph may not have had time to develop.

We thank Dr. E. W. L. Fletcher for reviewing the chest $x$-rays of the infants in this study; Mrs. D. Harris for her technical assistance; and Professor J. P. M. Tizard for his advice and criticism.

\section{REFERENCES}

Adams, F. H., Fujiwara, T., Emmanouilides, G., and Scudder, A. (1965). Surface properties and lipids from lungs of infants with hyaline membrane disease. Fournal of Pediatrics, 66, 357.

Avery, M. E., and Mead, J. (1959). Surface properties in relation to atelectasis and hyaline membrane disease. American fournal of Diseases of Children, 97, 517.

Avery, M. E., Gatewood, O. B., and Brumley, G. (1966). Transient tachypnoea of newborn. Possible delayed resorption of fluid at birth. American fournal of Diseases of Children, 111, 380.
Baum, J. D., and Roberton, N. R. C. (1974). Distending pressure in infants with respiratory distress syndrome. Archives of Disease in Childhood, 49, 771.

Blumenfeld, T. A., Driscoll, J. M., and James, L. S. (1974). Lecithin/sphingomyelin ratios in tracheal and pharyngeal aspirates in respiratory distress syndrome. Fournal of Pediatrics, 85, 403.

Borer, R. C., and Gluck, L. (1971). Lecithin/sphingomyelin ratio in amniotic fluid. Laboratory Schedule, Department of Pediatrics, University of San Diego, California, U.S.A.

Borer, R. C., Kuhns, L. R., Holt, J. F., Poznanski, A. K., and Bednarek, F. J. (1974). Accuracy of gastric aspirate lecithin/ sphingomyelin ratio and chest roentgenograms in the diagnosis of RDS. Pediatric Research, 8, 444.

Brumley, G. W., Hodson, W. A., and Avery, M. E. (1967). Lung phospholipids and surface tension correlations in infants with and without hyaline membrane disease and in adults. Pediatrics, 40, 13.

Chu, J., Clements, J. A., Cotton, E. K., Klaus, M. H., Sweet, A. Y., and Tooley, W. H. (1967). Neonatal pulmonary ischemia. Part 1, clinical and physiological studies. Pediatrics, 40, 709.

Dunn, P. M. (1974). Continuous positive airway pressure using the Gregory box. Proceedings of the Royal Society of Medicine, 67, 245.

Fox, H. A., Sarkozi, L., and Rosenfeld, W. N. (1974). Lecithin content in hypopharyngeal mucous of newborn infants. Pediatric Research, 8, 446.

Gluck, L., Kulovich, M. V., Borer, R. C., Brenner, P. H., Anderson, G. G., and Spellacy, W. M. (1971). Diagnosis of the respiratory distress syndrome by amniocentesis. American fournal of Obstetrics, and Gynecology, 109, 440.

Gluck, L., Kulovich, M. V., Borer, R. C., Keidal, W. N. (1974). The interpretation and significance of the lecithin/sphingomyelin ratio in amniotic fluid. American fournal of Obstetrics and Gynecology, 120, 142.

Gluck, L., Motoyama, E. K., Smits, H. L., and Kulovich, M. V. (1967). The biochemical development of surface activity in mammalian lung. I. The surface-active phospholipids; the separation and distribution of surface-active lecithin in the lung of the developing rabbit fetus. Pediatric Research, 1, 237.

Kanto, W. P., Borer, R. C., and Roloff, D. W. (1974a). Postnatal changes in the L/S ratio of tracheal aspirates (TA) from infants with severe respiratory distress syndrome (RDS). fournal of Pediatrics, 84, 921.

Kanto, W. P., Borer, R. C., Barr, M., and Roloff, D. W. (1974b). Tracheal aspirate (TA), lecithin/sphingomyelin ratio (LS) and recovery from RDS. Pediatric Research, 8, 447.

Krouskop, R. W., Brown, E. G., and Sweet, A. Y. (1974). The early use of constant positive airway pressure (CPAP) in hyaline membrane disease (HMD). Pediatric Research, 8, 448.

Lempert, J., and Macklem, P. T. (1971). Effect of temperature on rabbit lung surfactant and pressure-volume hysteresis. fournal of Applied Physiology, 31, 380.

Mockrin, L., and Bancalari, E. (1974). Continuous negative pressure in hyaline membrane disease: early versus late onset. Pediatric Research, 8, 448.

Schneider, P. B. (1966). Permanent sensitive stain for cholinecontaining phospholipids on thin-layer chromatograms. Fournal of Lipid Research, 7, 169.

Wagstaff, T. I., Whyley, G. A., and Freedman, G. (1974). The measurement of the lecithin/sphingomyelin ratio of amniotic fluid after thin-layer chromatography. Annals of Clinical Biochemistry, 11, 24.

Whitfield, C. R., and Sproule, W. B. (1974). Fetal lung maturation. British fournal of Hospital Medicine, 12, 768.

Correspondence to Dr. J. D. Baum, Department of Paediatrics, University of Oxford, John Radcliffe Hospital, Headington, Oxford OX3 9DU. 